EDITOR'S
CHOICE

\title{
Breathing exercises for asthma: a randomised controlled trial
}

\author{
M Thomas, ${ }^{1}$ R K McKinley, ${ }^{2}$ S Mellor, ${ }^{3}$ G Watkin, ${ }^{3}$ E Holloway, ${ }^{4}$ J Scullion, ${ }^{3}$ D E Shaw, ${ }^{3}$ \\ A Wardlaw, ${ }^{3}$ D Price, ${ }^{1}$ I Pavord ${ }^{3}$
}

- Additional information is given in the appendices published online only at http://thorax.bmj. com/content/vol64/issue1

${ }^{1}$ Department of General Practice and Primary Care, University of Aberdeen, Aberdeen, UK;

${ }^{2}$ Keele University Medical

School, Keele University, Keele, Staffordshire, UK; ${ }^{3}$ Institute for Lung Health, Glenfield Hospital, Leicester, UK; ${ }^{4}$ Department of Epidemiology and Public Health, University College London,

London, UK

Correspondence to:

Dr M Thomas, Department of General Practice and Primary

Care, University of Aberdeen,

Foresterhill Health Centre,

Westburn Road, Aberdeen AB25

2AY, UK; mikethomas@

doctors.org.uk

Received 28 April 2008

Accepted 15 September 2008

\section{ABSTRACT}

Background: The effect of breathing modification techniques on asthma symptoms and objective disease control is uncertain.

Methods: A prospective, parallel group, single-blind, randomised controlled trial comparing breathing training with asthma education (to control for non-specific effects of clinician attention) was performed. Subjects with asthma with impaired health status managed in primary care were randomised to receive three sessions of either physiotherapist-supervised breathing training $(n=94)$ or asthma nurse-delivered asthma education $(n=89)$. The main outcome was Asthma Quality of Life Questionnaire (AOLO) score, with secondary outcomes including spirometry, bronchial hyper-responsiveness, exhaled nitric oxide, induced sputum eosinophil count and Asthma Control Questionnaire (ACO), Hospital Anxiety and Depression (HAD) and hyperventilation (Nijmegen) questionnaire scores.

Results: One month after the intervention there were similar improvements in AOLO scores from baseline in both groups but at 6 months there was a significant between-group difference favouring breathing training (0.38 units, $95 \% \mathrm{Cl} 0.08$ to 0.68). At the 6-month assessment there were significant between-group differences favouring breathing training in $\mathrm{HAD}$ anxiety (1.1, $95 \% \mathrm{Cl} 0.2$ to 1.9$), \mathrm{HAD}$ depression $(0.8,95 \% \mathrm{Cl} 0.1$ to 1.4) and Nijmegen (3.2, 95\% Cl 1.0 to 5.4) scores, with trends to improved ACO $(0.2,95 \% \mathrm{Cl} 0.0$ to 0.4$)$. No significant between-group differences were seen at 1 month. Breathing training was not associated with significant changes in airways physiology, inflammation or hyper-responsiveness.

Conclusion: Breathing training resulted in improvements in asthma-specific health status and other patient-centred measures but not in asthma pathophysiology. Such exercises may help patients whose quality of life is impaired by asthma, but they are unlikely to reduce the need for anti-inflammatory medication.

Asthma is characterised by respiratory symptoms, variable airflow obstruction, airways inflammation and hyper-responsiveness. Although pharmacotherapy is effective for many patients, ${ }^{1}$ outcomes remain suboptimal ${ }^{2}$ for complex reasons including undertreatment and non-compliance. ${ }^{3}$ Many patients have concerns about regular medication, particularly with inhaled corticosteroids (ICS), ${ }^{4}$ and many use non-pharmacological and complementary therapies including breathing modification techniques. ${ }^{5}$ Although breathing exercises were formerly widely used, ${ }^{6}$ they are no longer part of mainstream asthma management following the introduction of effective pharmacotherapy.
There has recently been renewed interest in breathing techniques in asthma, fuelled partially by alternative techniques such as the Butekyo breathing method ${ }^{7-9}$ and yogic breathing. ${ }^{10} 11$

Physiotherapy-based breathing modification studies have reported improvements in quality of life ${ }^{1213}$ and reductions in bronchodilator $u^{14}$ in asthma. A Cochrane review of breathing exercises for asthma concluded that there were trends to improvement, but the current evidence was inadequate. ${ }^{15}$ Hypocapnia ${ }^{16}$ and symptoms of dysfunctional breathing ${ }^{17}$ may be more prevalent in subjects with asthma than in the general population, ${ }^{18}$ so breathing modification may treat epiphenomena and associated co-morbidities rather than asthma per se.

We hypothesised that breathing retraining would improve asthma health status and asthma control without changing objective physiological and inflammatory markers.

\section{METHODS}

\section{Setting and participants}

The effects of three sessions of physiotherapistdirected breathing exercises were compared with a "control" of three sessions of nurse-provided asthma education. Patients treated for asthma in 10 UK primary care general practices in Leicester, UK and having moderate impairment of asthmarelated health status (Asthma Quality of Life Questionnaire score <5.5) were recruited (see details in online supplement). Blinding was impossible for participants but data entry and analysis were performed blind to randomisation status. Usual physicians were requested to maintain baseline therapy during the study if possible.

\section{Clinical methods}

At the baseline and 1 month post-intervention study visits the following measurements were made:

- Asthma Quality of Life Questionnaire (AOLO). ${ }^{19}$

- Asthma Control Questionnaire (ACQ). ${ }^{20}$

- Nijmegen hyperventilation questionnaire (NQ). ${ }^{21}$

- Hospital Anxiety and Depression (HAD) questionnaire. ${ }^{22}$

- Spirometric values and bronchodilator reversibility.

- Resting minute volume (MV).

- Resting end tidal carbon dioxide concentration $\left(\mathrm{ETCO}_{2}\right)$ 
- Bronchial hyper-responsiveness (concentration of methacholine required to provoke a $20 \%$ fall in the forced expiratory volume in $\left.1 \mathrm{~s}\left(\mathrm{FEV}_{1}\right), \mathrm{PC}_{20}\right)$.

- Induced sputum differential cell count analysis.

- Fraction of exhaled nitric oxide at a flow of $50 \mathrm{ml} / \mathrm{s}$ (FenO; Aerocrine Nioxx analyser).

Laboratory measurements were performed in the same order and at the same time of day at each assessment, with FenO measured first.

Questionnaires were mailed to subjects 6 months after the intervention.

\section{Intervention}

Subjects were randomised to either breathing training (BT) or control groups. Study attendances for both groups consisted of three sessions, an initial 60 min small group session (2-4 subjects) followed by two individual sessions of $30-45 \mathrm{~min}$ with 2-4 weeks between attendances.

In the BT group, explanation of normal breathing and possible effects of abnormal "dysfunctional breathing" such as over-breathing, mouth breathing and upper chest breathing was provided. In individual sessions, subjects were taught appropriate regular diaphragmatic and nasal breathing techniques (similar to the Papworth method ${ }^{13}$ ) and encouraged to practise these exercises for at least $10 \mathrm{~min}$ each day. We controlled for non-specific effects of professional attention by allocating similar sessions with a health professional (asthma nurse) delivering asthma education. This intervention comprised information on the nature of asthma followed by individual sessions, presenting broad asthma and atopy concepts and explaining treatment rationale without providing personalised asthma advice. ${ }^{23}$

\section{Statistical analysis}

As we were interested in the immediate and longer-term impact of the intervention, the primary outcome was a comparison between groups of AOLO changes from baseline values measured and independently analysed 1 and 6 months after the intervention. Secondary outcomes were changes in ACO scores, HAD scores, respiratory physiology, FenO and sputum eosinophila. Within-group comparisons were examined using a paired $t$ test or Wilcoxon test, while between-group comparisons were made using the independent sample $t$ test or the Mann-Whitney test. Pearson correlation coefficients assessed association between two continuous variables. Analyses were performed on an "intention to treat" basis with "per protocol" sensitivity analyses performed on subjects completing measurements (see online supplement for further details). We also assessed whether the NQ (a screening tool for symptomatic hyperventilation) score $(<23$ or $\geqslant 23$ ) or physiological evidence of hyperventilation influenced the response to breathing retraining.

\section{RESULTS}

Invitations were sent to all 3139 adult patients with asthma in the participating centres, 516 of whom replied expressing

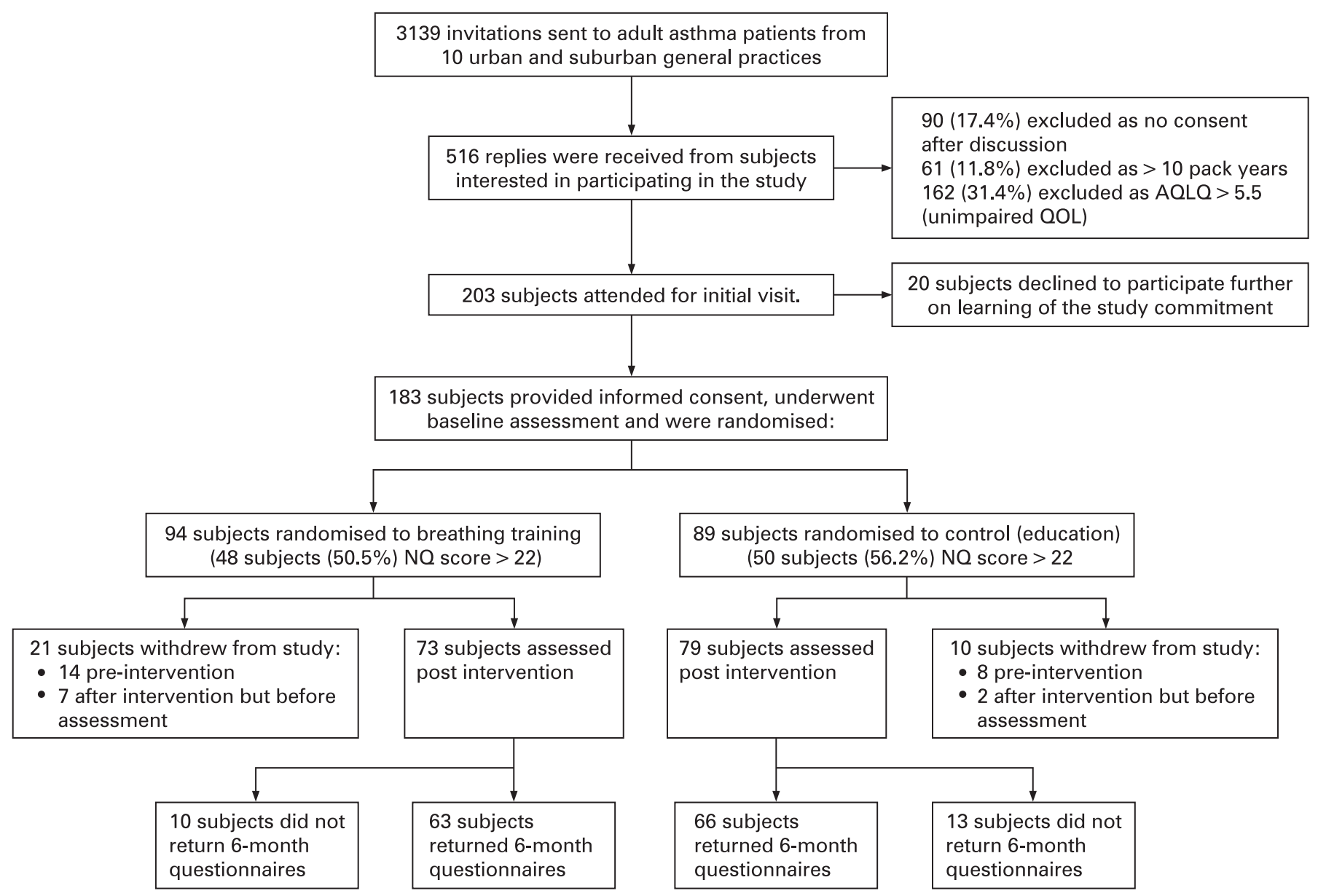

Figure 1 Study flow diagram. AQLO, Asthma Quality of Life Questionnaire; NQ, Nijmegen hyperventilation questionnaire; Q0L, quality of life. 
Table 1 Baseline demographic characteristics of study subjects

\begin{tabular}{|c|c|c|}
\hline & $\begin{array}{l}\text { Breathing training } \\
(\mathrm{n}=94 \text { if not stated) }\end{array}$ & $\begin{array}{l}\text { Control } \\
\text { ( } n=89 \text { if not stated) }\end{array}$ \\
\hline Age (years) ${ }^{*}$ & $46.0(33.0-57.3)$ & $46.0(35.0-57.0)$ \\
\hline Female & $52(58.4 \%)$ & $60(63.8 \%)$ \\
\hline AQLO score $\dagger$ & $4.2(0.9)$ & $4.3(1.0)$ \\
\hline Body mass index* & $27.7(24.0-31.3)$ & $25.7(23.0-29.2)$ \\
\hline ACQ score $\dagger$ & $1.4(0.8)$ & $1.5(0.9)$ \\
\hline NQ score $\dagger$ & $24.1(9.4)$ & $23.2(8.4)$ \\
\hline$\%$ Predicted $\mathrm{FEV}_{1} \dagger$ & $87.3(18.8)$ & $91.8(21.6)$ \\
\hline Bronchodilator reversibility $(\%)^{*}$ & $5.5(2.2-10.2), n=74$ & $5.7(3.5-12.2), \mathrm{n}=77$ \\
\hline $\begin{array}{l}\text { ICS dose ( } \mu \mathrm{g} / \text { day BDP } \\
\text { equivalent) }\end{array}$ & $400(0-525)$ & $400(113-600)$ \\
\hline LABA treatment & $43(46.3 \%)$ & $36(40.4 \%)$ \\
\hline LTRA treatment & $3(3.2 \%)$ & $2(2.3 \%)$ \\
\hline $\begin{array}{l}\text { Oral steroid burst in previous } \\
\text { year }\end{array}$ & $29(30.5 \%)$ & $19(21.3 \%)$ \\
\hline $\begin{array}{l}\text { Average daily SABA use } \\
\text { (doses/day) } \dagger\end{array}$ & $1.39(1.28)$ & $1.47(1.42)$ \\
\hline \multicolumn{3}{|l|}{ BTS treatment step } \\
\hline Step 1 & $20(21.3 \%)$ & $20(22.5 \%)$ \\
\hline Step 2 & $37(39.4 \%)$ & $34(38.2 \%)$ \\
\hline Step 3 & $35(37.2 \%)$ & $34(38.2 \%)$ \\
\hline Step 4 & $2(2.1 \%)$ & $1(1.1 \%)$ \\
\hline Rhinitis/hay fever & $58(61.1 \%)$ & $55(61.8 \%)$ \\
\hline HAD anxiety score $\dagger$ & $7.2(3.8)$ & $7.5(4.0), n=88$ \\
\hline HAD depression score $\dagger$ & $3.3(3.0)$ & $3.6(2.9), n=88$ \\
\hline
\end{tabular}

* Median (interquartile range). $†$ Mean (SD).

ACQ, Asthma Control Questionnaire; AOLQ, Asthma Quality of Life Questionnaire; BDP, beclomethasone dipropionate; BTS, British Thoracic Society; FEV 1 , forced expiratory volume in $1 \mathrm{~s} ; \mathrm{HAD}$, Hospital Anxiety and Depression Questionnaire; ICS, inhaled corticosteroids; LABA, long-acting $\beta_{1}$ agonist; LTRA, leukotriene receptor antagonist; NQ, Nijmegen Questionnaire; SABA, short-acting $\beta_{1}$ agonist.

interest. Two hundred and twenty-three did not meet the entry criteria (162 unimpaired quality of life, 61 smokers) and 110 declined to participate on receiving more information (fig 1). One hundred and eighty-three subjects provided informed consent, underwent baseline evaluation and were randomised to the BT $(n=94)$ or control $(n=89)$ group (fig 1$)$. Baseline clinical (table 1) and physiological (table 2) characteristics were similar between the groups. Twenty-two subjects $(12.0 \% ; 14$ BT and 8 control subjects) failed to attend for the intervention sessions and took no further part in the study. Nine subjects (4.9\%; 7 BT and 2 control subjects) attended one or more intervention sessions but did not attend for 1-month follow-up assessments. Six-month postal questionnaires were not returned by a further 23 subjects $(12.6 \% ; 10$ BT and 13 control subjects). The withdrawal rate was not significantly different between the groups at the pre-intervention $(p=0.26)$, 1-month post-intervention $(p=0.27$ ) or 6 -months post-intervention time points ( $p=0.83$ ). Withdrawals occurred due to inconvenience of attending visits or lack of motivation to continue participation in the study.

Changes in ICS dose occurred in 33 subjects (16 BT, 17 control subjects), with median (interquartile range) change of 100 ( -300 to 250$) \mu \mathrm{g} /$ day beclomethasone equivalent, with no significant difference between groups $(p=0.22)$.

\section{Primary outcome: asthma-related quality of life}

Significant improvements in AOLO scores occurred following intervention in both the BT and control groups, with no significant between-group differences at the 1-month assessment (fig 2). The mean ( $95 \%$ confidence interval, CI) change in
AOLO score was 0.92 (0.71 to 1.22) units in the BT group and 0.88 (0.66 to 1.10$)$ in the control group, with a between-group difference of $0.04(-0.26$ to $0.34, p=0.78)$. However, at the 6month assessment the improvements from baseline in the BT group was 1.12 (0.92 to 1.32) compared with a smaller change of 0.74 (0.51 to 0.97 ) in the control group, with a significant between-groups difference of 0.38 ( 0.08 to $0.68, p=0.01)$. In the per protocol analysis, a similar but more marked picture emerged, with no significant differences between groups at 1 month but a larger difference at 6 months $(0.64,0.28$ to 1.01 , $\mathrm{p}=0.001)$.

With a change of 0.5 signifying a clinically relevant individual patient threshold, ${ }^{24}$ a higher proportion of the BT group than the control group showed a clinically important improvement in AOLQ score at the 6-month evaluation in intention to treat $(71.3 \%$ vs $56.2 \%, p=0.03)$ and in per protocol $(90.5 \%$ vs $63.6 \%$, $\mathrm{p}<0.001)$ analyses. The number needed to treat ${ }^{25}$ for a subject in the BT group to improve over a control subject was 5.6 (3.6 on per protocol analysis) at the 6-month assessment.

When analysed by the four subdomains of the AOLO (table 3), similar improvements in scores were seen at the 1-month assessment in both groups with no significant intergroup differences, but at 6 months significantly greater improvements were found in the intervention group in the symptoms $(p=0.01)$, activities $(p=0.01)$ and emotions $(p=0.05)$ domains but not in the environment domain $(p=0.40)$.

\section{Secondary outcomes: pulmonary physiology}

Methacholine $\mathrm{PC}_{20}$ was assessed at baseline in 90/95 BT and 87/ 89 control subjects (failures due to contraindications or lack of consent) and at 1 month post-intervention in all BT subjects $(n=73)$ and $78 / 79$ control subjects. $\mathrm{PC}_{20}$ did not change significantly from baseline in either group (mean change 0.29 ( $95 \%$ CI -0.13 to 0.73 ) doubling doses in BT group ( $p=0.19)$ and 0.09 (95\% CI -0.20 to 0.57 ) doubling doses in control group $(p=0.72)$, table 2$)$. The between-group difference was 0.02 (95\% CI -0.04 to 0.09$)$ doubling doses $(p=0.54)$.

Small increases in prebronchodilator $\mathrm{FEV}_{1}$ were observed in both groups with a non-significant trend favouring the control group (mean between-group difference -0.0631 (95\% CI -0.130 to 0.004$), p=0.07$, table 2 ).

MV was significantly reduced in both groups with no significant intergroup difference (mean difference -0.571 (95\% CI -2.20 to 1.05$), p=0.49$ ). $\mathrm{ETCO}_{2}$ did not change significantly within or between groups (mean difference $0.08 \mathrm{kPa}(95 \% \mathrm{CI}-0.15$ to 0.30$), \mathrm{p}=0.51)$.

Sputum induction for differential cell estimation was successful at baseline in 76/95 BT subjects and 75/89 control subjects and in 66/73 BT subjects and $62 / 79$ control subjects at the outcome visit, with failures due to contraindications, lack of consent or inadequate sample production after induction. The differential eosinophil count did not change significantly in either group (table 2), with no significant difference between groups (mean between-group difference -0.20 fold change $(95 \%$ CI -0.63 to 0.23 ), $p=0.35$ ).

Baseline FeNO concentration was measured in 94/95 BT subjects and $87 / 89$ control subjects (with missing values due to failure to perform an adequate expiratory effort) and on all attendees at follow-up. A significant reduction in FeNO was found in the control group but not in the BT group (table 2), although no significant difference was found between groups (mean difference $-5.3 \mathrm{ppb}$ (95\% CI -19.0 to 8.4), $\mathrm{p}=0.44$ ). 
Table 2 Change in physiological outcome parameters in breathing training and control groups between the baseline and outcome (1 month) assessments in the intention to treat population

\begin{tabular}{|c|c|c|c|c|c|c|c|c|}
\hline & \multicolumn{3}{|c|}{ Breathing training group } & \multicolumn{3}{|l|}{ Control group } & \multirow[b]{2}{*}{$\begin{array}{l}\text { Mean }(95 \% \mathrm{CI}) \\
\text { between-group difference }\end{array}$} & \multirow[b]{2}{*}{ p Value } \\
\hline & Baseline & $\begin{array}{l}1 \text { month } \\
\text { post-intervention }\end{array}$ & p Value & Baseline & $\begin{array}{l}1 \text { month } \\
\text { post-intervention }\end{array}$ & p Value & & \\
\hline $\mathrm{FEV}_{1}(\mathrm{I})^{*}$ & $2.85(0.83)$ & $2.95(0.83)$ & $<0.001$ & $2.82(0.76)$ & $2.97(0.78)$ & $<0.001$ & $-0.06(-0.13$ to 0.00$)$ & 0.07 \\
\hline Feno $(p p b) \dagger$ & $25.5(21.7$ - to 29.9$)$ & 23.7 (20.0 to 28.2$)$ & 0.14 & 30.5 (25.6 to 36.3$)$ & $26.8(22.6$ to 31.8$)$ & 0.02 & $5.3(-8.4$ to 19.0$)$ & 0.44 \\
\hline $\begin{array}{l}\text { Sputum } \\
\text { eosinophils }(\%) \dagger\end{array}$ & 1.21 (0.89 to 1.65$)$ & 1.21 (0.85 to 1.72$)$ & 0.97 & 1.88 (1.27 to 2.27 ) & 1.52 (1.06 to 2.18$)$ & 0.28 & $-0.20(-0.63$ to 0.23$) \S$ & 0.35 \\
\hline MV $(I) \dagger$ & $13.49(6.07)$ & $11.21(4.57)$ & $<0.001$ & $13.54(5.44)$ & $11.84(4.82)$ & $<0.005$ & $-0.57(-2.20$ to 1.05$)$ & 0.49 \\
\hline
\end{tabular}

*Mean (SD). †Geometric mean (transformed 95\% confidence interval). \$Doubling doses. §Fold change.

$\mathrm{ETCO}_{2}$, end tidal carbon dioxide; Feno, fraction of exhaled nitric oxide; $\mathrm{FEV}_{1}$, forced expiratory volume in $1 \mathrm{~s}$; $\mathrm{MV}_{\text {, minute volume; }} \mathrm{PC}_{20}$, concentration of inhaled methacholine provoking a $20 \%$ fall in $\mathrm{FEV}_{1}$.

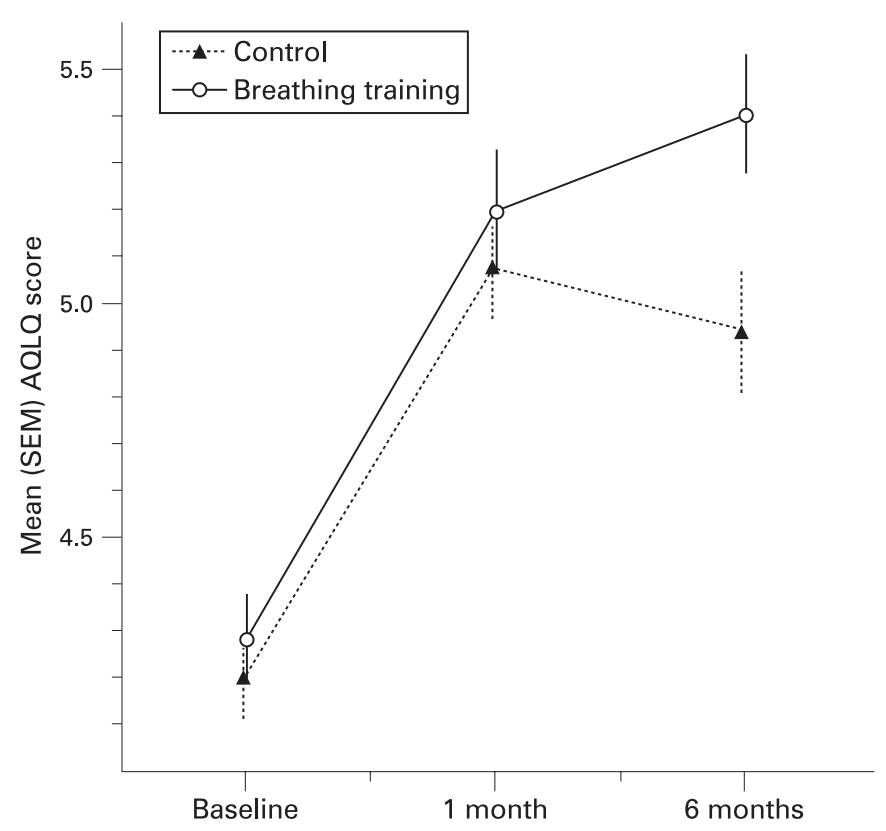

Figure 2 Mean (SEM) Asthma Quality of Life Questionnaire (AOLO) scores in breathing training and control groups at baseline and at 1 and 6 months post-intervention in intention to treat population (increased score equates to better quality of life).

\section{Secondary outcomes: questionnaires}

Significant improvements in ACQ were observed in both groups with no significant differences between groups at the 1-month assessment (mean difference 0.05 (95\% CI -0.16 to 0.25 ), $\mathrm{p}=0.70$, table 4). At the 6-month assessment, non-significant trends favouring the BT group were seen (between-group difference -0.17 (95\% CI -0.38 to 0.04$), p=0.12$, fig 3$)$. The between-group difference was significant in the per protocol analysis $(-0.29(95 \% \mathrm{CI}-0.57$ to -0.01$), p=0.04$, see online supplement).

There were significant reductions in the HAD Anxiety and Depression domain scores in both groups 1 month following the intervention with no significant difference between the groups (table 4). At the 6-month assessment significant between-group differences were observed favouring the BT group in the Anxiety score (mean difference -1.05 (95\% CI -1.94 to $-0.16), p=0.02)$ and the Depression score $(-0.75$ (95\% CI -1.40 to -0.10$), p=0.03)$. Similarly, reductions in the $\mathrm{NQ}$ score were not significantly different between groups at 1 month but by 6 months the difference favoured the BT group (mean difference -3.16 (95\% CI -5.35 to -0.97 ), $\mathrm{p}=0.005$ ).

Subjects reported reduced average daily bronchodilator use from the baseline to 1-month assessments in both groups with no significant differences between the groups (difference -0.06 (95\% CI -0.36 to $0.25, \mathrm{p}=0.72$ ).

\section{Influence of hyperventilation markers on response to breathing training}

In subjects undergoing BT, no significant difference was observed in the change from baseline AOLO score between the subgroup with a $\mathrm{NQ}$ score of $\geqslant 23$ (suggesting symptomatic hyperventilation) and those with a score of $<23$ at the 1 -month $(p=0.28)$ or the 6-month $(p=0.66)$ assessments (see table E5 in online supplement). Significant correlations were observed between changes in AOLO and changes in NQ scores at both time points, but similar correlations were also seen in the control group (see table E3 in online supplement).

Improvements in AOLO scores were not significantly different between subjects with hypocapnia at the baseline assessment (lowest quartile $\mathrm{ETCO}_{2}$ ) and the remaining subjects

Table 3 Mean (95\% CI) change in Asthma Quality of Life Questionnaire (AOLO) score from baseline readings at 1 and 6 months post-intervention in breathing training and control groups

\begin{tabular}{|c|c|c|c|c|c|c|c|c|}
\hline & \multicolumn{4}{|l|}{1 month } & \multicolumn{4}{|l|}{6 months } \\
\hline & $\begin{array}{l}\text { Breathing training } \\
(\mathrm{n}=94)\end{array}$ & $\begin{array}{l}\text { Control } \\
(\mathrm{n}=89)\end{array}$ & $\begin{array}{l}\text { Between-group } \\
\text { difference }\end{array}$ & p Value & $\begin{array}{l}\text { Breathing training } \\
(\mathrm{n}=94)\end{array}$ & $\begin{array}{l}\text { Control } \\
(\mathrm{n}=89)\end{array}$ & $\begin{array}{l}\text { Between-group } \\
\text { difference }\end{array}$ & p Value \\
\hline Total & 0.92 (0.71 to 1.22$)$ & $0.88(0.66$ to 1.10$)$ & $0.04(-0.26$ to 0.34$)$ & 0.78 & $1.12(0.92$ to 1.32$)$ & $0.74(0.51$ to 0.97$)$ & $0.38(0.08$ to 0.68$)$ & 0.01 \\
\hline Activities & 0.82 (0.61 to 1.02 ) & $0.71(0.46$ to 0.97$)$ & $0.10(-0.22$ to 0.43$)$ & 0.53 & $0.92(0.71$ to 1.13$)$ & $0.48(0.19$ to 0.76$)$ & $0.44(0.09$ to 0.80$)$ & 0.01 \\
\hline Emotion & 1.11 (0.87 to 1.35$)$ & $1.18(0.86$ to 1.50$)$ & $-0.07(-0.46$ to 0.32$)$ & 0.72 & 1.29 (1.04 to 1.54$)$ & 0.870 .56 to 1.22 ) & $0.40(0$ to 0.81$)$ & 0.05 \\
\hline Environment & 0.81 (0.58 to 1.03 ) & 0.91 (0.64 to 1.18$)$ & $-0.10(-0.46$ to 0.25$)$ & 0.56 & $0.97(0.74$ to 1.20$)$ & $0.82(0.55$ to 1.09$)$ & $0.15(-0.20$ to 0.50$)$ & 0.40 \\
\hline
\end{tabular}

Total AQLO score and individual AQLO domains in the intention to treat population. 


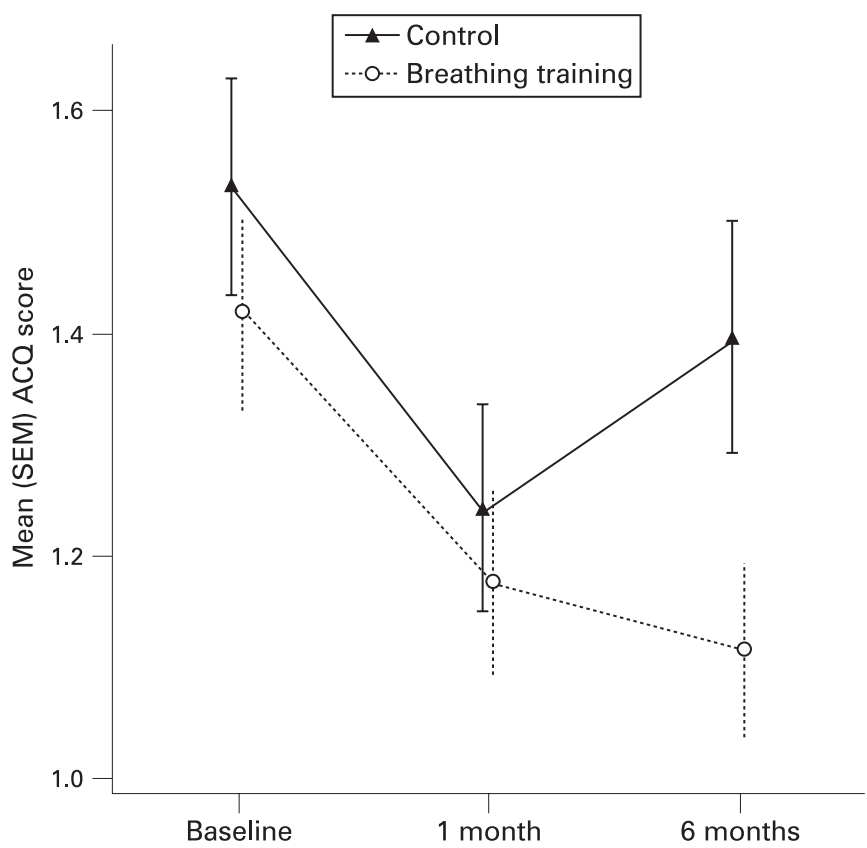

Figure 3 Mean (SEM) Asthma Control Questionnaire (ACQ) scores in breathing training and control groups at baseline and at 1 and 6 months post-intervention in intention to treat population (decreased score relates to reduced symptoms).

at the 1-month assessment (mean between-group difference $0.17(95 \%$ CI -0.31 to 0.66$), p=0.47)$ or the 6 -month assessment $(0.15(95 \%$ CI -0.33 to 0.63$), p=0.53)$. No significant correlations were observed between baseline $\mathrm{ETCO}_{2}$ or MV and change in AOLO scores, but a weak correlation was observed between a reduction in MV and improvements in AOLO scores (Pearson correlation coefficient $-0.24, \mathrm{p}=0.02$ ).

\section{DISCUSSION}

This study investigated the hypothesis that BT exercises have specific effects on asthma control. A brief physiotherapistsupervised breathing retraining programme was found to be associated with improvements in the primary outcome (asthma-related health status) above those seen in the control arm at the 6-month assessment. At the early post-intervention assessment, the control group (who received nurse-delivered asthma education involving similar professional contact time) was associated with similar improvements in health status from baseline values to those observed in the BT group; these withingroup improvements could be explained by spontaneous improvement in symptomatic patients (regression to the mean effects) by non-specific trial involvement and professional attention effects, by specific improvements resulting from the control and breathing interventions, or by combinations of these factors. At the 6-month assessment a significant difference was observed between the groups, confirming a specific benefit associated with the breathing programme independent of potentially confounding factors. The magnitude of the changes in health status was considerable; the mean improvement from baseline in the BT and control groups at the 6-month assessment were 1.1 and 0.7 , respectively, with a change of 0.5 being the clinically important threshold for change in an individual patient. ${ }^{24}$ The mean between-group difference was 0.4 and the number needed to treat for one extra patient to achieve a clinically relevant improvement in the breathing group 
over the control group ${ }^{25}$ was 6 . The threshold of clinical relevance for between-group mean differences in AQLO scores has not been established but, for comparison, the final betweengroup differences reported between the fluticasone monotherapy and the salmeterol-fluticasone arms in the GOAL study ${ }^{1}$ in which subjects had similar baseline demographic profiles and AOLO scores-was between 0.1 and 0.3 in the different strata (so considerable less than the between-group differences seen in our study), and the overall improvements from baseline to the end of the step-up phase (phase 1) in subjects receiving fluticasone ranged between 0.8 and 1.3 , similar in magnitude to the changes from baseline to the 6-month evaluation in patients undergoing BT.

Controlling for complex interventions is methodologically difficult but it is necessary to provide a comparable control procedure that is credible and acceptable to patients as, without an adequate control process, it cannot safely be assumed that resulting clinical benefits relate to specific factors in the intervention rather than to spontaneous improvements, the effects of trial involvement or non-specific placebo-like effects resulting from attention from a healthcare professional. A recent study investigating a similar breathing modification programme against a comparison control group receiving "usual care" reported improved asthma-related health status, symptoms and mood in adult patients with asthma undergoing BT, ${ }^{13}$ with little change in the control group. However, it is well established that professional attention of itself can result in beneficial non-specific "placebo-like" effects, ${ }^{26-28}$ and it has previously been suggested that breathing exercises are in effect an elaborate placebo. ${ }^{29}$ The present study therefore aimed to provide a "neutral" control by giving the subjects randomised to the control arm equal time with a healthcare professional (a trained asthma nurse) in a similar environment. The nurse provided generic non-personalised asthma information without changing medication or providing personalised self-management advice but, in the context of this study (with subjects having impaired asthma-related health status recruited from the community), the control group was associated with improvements from baseline in all patient-centred outcomes and, indeed, a small but statistically significant reduction in FeNO concentrations implying a possible reduction in airways inflammation. The provision of a personalised asthma action plan including asthma education has been shown to improve outcomes, ${ }^{30}$ although little effect has previously been reported from limited information provision alone. ${ }^{23}$ In view of the improvement in health status from baseline in this group and the lack of any suggestion that educational initiatives may result in a deterioration in health status, it is very unlikely that the between-group difference noted was a result of a negative effect in the control arm; indeed, it is possible that the effects of BT are underestimated because of a beneficial effect of the educational programme. Future studies should investigate whether benefits from education and breathing exercises may be additional and synergistic.

The high number of subjects dropping out of the study before the interventions (12\%) may have diluted the between-group differences, and in the per protocol analyses the between-group difference at 6 months was 0.6 and the number needed to treat was 4 . In parallel with the persistence in improvements in the overall AOLO scores, consistent improvements were seen in the BT group compared with the control group at the 6-month assessment but not at the 1-month assessments in three of the four subdomains of the health status instrument (symptoms, activities and emotions) and in other outcomes measuring the patients' personal experience of their condition, including anxiety and depression scores. Similarly, symptomatic asthma control (ACQ score) improved initially in both groups but showed better persistence in the BT group. Breathing exercises were not associated with significant changes in airways inflammation or hyper-responsiveness so, although associated with beneficial effects on the patients' experience of their illness, they are unlikely to modify the pathophysiology of asthma.

Asthma-related health status was selected as the primary end point in this study as this parameter best measures patients' experience of asthma and the effect of the condition on their daily lives. ${ }^{19}{ }^{31}$ Asthma is a complex and multifaceted condition, and dissociations have been described between health status and other asthma control parameters, ${ }^{32}{ }^{33}$ with asthma quality of life being shown to be an independent measure of control that does not correlate closely with other control measures. ${ }^{31}$ In this study we measured a range of outcome parameters and found that breathing exercises were consistently associated with improvement in "patient-centred" measures such as health status, symptoms and psychological measures, but not with changes in physiological or biomarker parameters of asthma control. Breathing exercises were associated with a non-significant increase in $\mathrm{ETCO}_{2}$ and a significant reduction in $\mathrm{MV}$, implying that hypoventilation was to some extent achieved. However, a reduction in $\mathrm{MV}$ also occurred in the control group, without significant differences between groups, suggesting that this was a non-specific finding. These data do not strongly support the hypothesis that reducing hyperventilation is the primary mechanism by which improvements in health status are achieved with breathing retraining. This study did not address the regularity of breathing or the pattern of respiratory excursion. Previous studies have reported that hyperinflation, respiratory instability, upper chest and asynchronous breathing patterns can be associated with symptomatic dysfunctional breathing, ${ }^{34}$ and the effects of BT on these measures warrants attention. The effects of breathing exercises were similar in those with positive and negative screening scores on the Nijmegen hyperventilation questionnaire, so this instrument does not identify those who potentially benefit from the intervention; similarly, no correlation was seen between baseline $\mathrm{ETCO}_{2}$ or $\mathrm{MV}$ readings and the response to breathing exercises, or between changes in these parameters and changes in outcome measures (see online supplement), implying that the effects of breathing exercises were not mediated through reductions in hypocapnia or hyperventilation. Improvements in anxiety and depression scores were greater in the breathing group at the 6-month assessment, so part of the effect may have arisen through improvements in psychological well-being.

Subjects with mild to moderate symptomatic asthma were recruited from primary care settings using deliberately broad entry criteria. In any intervention study it cannot be assumed that the population studied is typical of the at-risk population, but we feel this study is likely to have recruited subjects representative of the wider population with mild to moderate asthma so the results are likely to be generalisable, which is not always true of asthma clinical trials, ${ }^{35}$ although it remains possible that subjects responding to the study invitation were in some way more sympathetic and susceptible to such approaches. The study had a fairly high dropout rate, although it should be noted that less volunteer-demanding pharmacological studies also report high dropout rates (eg, 15\% in the GOAL study ${ }^{1}$ ). Recruitment and retention are problems in community-based asthma studies, particularly with studies in a population with mild to moderate asthma, a typical clinical 
population, and the protocol involves disruption to patients' lives by additional medical visits and measurements. The dropout rate was, however, similar between the two groups and was highest at the start of the study where $11 \%$ of randomised subjects left the study before any of the intervention visits had occurred, so they were not in any way exposed to the effects of BT or the educational control. The similar (but more pronounced) between-group differences seen in the per protocol analyses support the overall messages from the study.

In summary, this randomised controlled trial found that adults with asthma taught breathing exercises showed improvements in health status, symptoms and psychological well-being after 6 months compared with those receiving an asthma education programme. Breathing exercises did not alter objective measures of airway hyper-responsiveness or inflammation, so cannot replace the need for anti-inflammatory medication. This study suggests that breathing exercises may potentially have a role in patients with suboptimally controlled mild to moderate asthma, but the use of such techniques must occur with patient education on the ongoing need for anti-inflammatory pharmacotherapy.

Acknowledgements: The authors thank Professors A Lee and P Hannaford for review and advice on the statistical methodology; Professor $E$ Juniper for advice on the analysis of the questionnaire data and comment on the manuscript; the patients and staff of the Leicester general practices who participated in the study; the staff of the respiratory function laboratory, Glenfield Hospital (particularly D D Vara and B Hargendon) for help with the physiological testing; $\mathrm{H}$ Watkinson for data entry; and Mike Berry for constructing the Access database.

Funding: This study was funded by a grant from Asthma UK (03/014). MT is in receipt of an Asthma UK Senior Research Fellowship.

Competing interests: None.

Ethics approval: Ethical approval was provided by the Leicestershire research ethics committee.

\section{REFERENCES}

1. Bateman ED, Boushey H, Busse WW, et al. Can guideline-defined asthma control be achieved? Am J Respir Crit Care Med 2004;170:836-44.

2. Rabe KF, Vermeire PA, Soriano JB, et al. Clinical management of asthma in 1999: the Asthma Insights and Reality in Europe (AIRE) study. Eur Respir J 2000;16:802-7.

3. Williams LK, Pladevall M, Xi H, et al. Relationship between adherence to inhaled corticosteroids and poor outcomes among adults with asthma. J Allergy Clin Immunol 2004;114:1288-93.

4. Apter AJ, Reisine ST, Affleck G, et al. Adherence with twice-daily dosing of inhaled steroids: socioeconomic and health-belief differences. Am J Respir Crit Care Med 1998;157:1810-7.

5. Slader CA, Reddel HK, Jenkins CR, et al. Complementary and alternative medicine use in asthma: who is using what? Respirology 2006;11:373-87.

6. Osler W. The principles and practice of medicine. Edinburgh: Y J Pentland, 1892.

7. Stalmatski A. Freedom from asthma: Butekyo's revolutionary treatment. London: Kyle Cathie Ltd, 1997.

8. Opat AJ, Cohen MM, Bailey MJ, et al. A clinical trial of the Buteyko Breathing Technique in asthma as taught by a video. J Asthma 2000;37:557-64.
9. Bowler SD, Green A, Mitchell CA. Butekyo breathing techniques in asthma: a blinded randomised controlled trial. Med J Aust 1998;169:575-8.

10. Cooper S, Oborne J, Nelson S, et al. The effect of two breathing exercises (Butekyo and pranayama) in asthma: a randomised controlled trial. Thorax 2003;58:674-9.

11. Manocha R, Marks G, Kenchington P, et al. Sahaja yoga in the management of moderate to severe asthma: a randomised controlled trial. Thorax 2002;57:110-5.

12. Thomas $\mathbf{M}$, McKinley RK, Freeman $\mathbf{E}$, et al. Breathing retraining for dysfunctional breathing in asthma: a randomised controlled trial. Thorax 2003;58:110-5.

13. Holloway E, West R. Integrated breathing and relaxation training (the Papworth Method) for adults with asthma in primary care: a randomised controlled trial. Thorax 2007:62:1033-4.

14. Slader CA, Reddel HK, Spencer LM, et al. Double blind randomised controlled trial of two different breathing techniques in the management of asthma. Thorax 2006;61:643-5.

15. Holloway E, Ram FSF. Breathing exercises for asthma. Cochrane Database System Rev 2004;(1):CD001277.

16. Osborne CA, O'Connor BJ, Lewis A, et al. Hyperventilation and asymptomatic chronic asthma. Thorax 2000;55:1016-22.

17. Thomas M, McKinley RK, Freeman E, et al. Prevalence of dysfunctional breathing in patients treated for asthma in primary care: cross sectional survey. BMJ 2001;322:1098-100.

18. Thomas M, McKinley RK, Freeman E, et al. The prevalence of dysfunctional breathing in adults in the community with and without asthma. Prim Care Respir $J$ 2005; 14:78-82.

19. Juniper EF, Guyatt GH, Cox FM, et al. Development and validation of the Mini Asthma Quality of Life Questionnaire. Eur Respir J 1999;14:32-8.

20. Juniper EF, O'Byrne PM, Guyatt GH, et al. Development and validation of a questionnaire to measure asthma control. Eur Respir J 1999;14:902-7.

21. van Dixhoorn J, Duivenvoorden HJ. Efficacy of Nijmegen questionnaire in recognition of the hyperventilation syndrome. J Psychosom Res 1985;29:199-206.

22. Snaith P, Zigmond AS, Snaith RP. Anxiety and depression in general medical settings. The hospital anxiety and depression scale. BMJ 1988;297:1544.

23. Gibson PG, Powell H, Coughlan J, et al. Limited (information only) patient education programs for adults with asthma. Cochrane Database System Rev 2002; (1):CD001005

24. Juniper EF, Guyatt GH, Willan A, et al. Determining a minimal important change in a disease-specific quality of life questionnaire. J Clin Epidemiol 1994;47:81-7.

25. Guyatt $\mathbf{G H}$, Juniper EF, Walter SD, et al. Interpreting treatment effects in randomised trials. BMJ 1998;316:690-3.

26. Crow R, Gage H, Hampson $\mathrm{S}$, et al. The role of expectancies in the placebo effect and their use in the delivery of health care: a systematic review. Health Technol Assess 1999;3:1-96.

27. Mason S, Tovey P, Long AF. Evaluating complementary medicine: methodological challenges of randomised controlled trials. BMJ 2002;325:832-4.

28. Patterson C, Dieppe P. Characteristic and incidental (placebo) effects in complex interventions such as acupuncture. BMJ 2005;330:1202-5.

29. Garssen B. Breathing retraining: a rational placebo? Clin Psychol Rev 1992:12:141-53.

30. Gibson PG, Powell H, Coughlan J, et al. Self-management education and regular practitioner review for adults with asthma. Cochrane Database System Rev 2002;(3):CD001117.

31. Juniper EF, Wisniewski ME, Cox FM, et al. Relationship between quality of life and clinical status in asthma: a factor analysis. Eur Respir J 2004;23:287-91.

32. Moy ML, Israel E, Weiss ST, et al, NHBLI Asthma Clinical Research Network. Clinical predictors of health-related quality of life depend on asthma severity. Am J Respir Crit Care Med 2001;163:924-9.

33. Rimington LD, Davies DH, Lowe D, et al. Relationship between anxiety, depression, and morbidity in adult asthma patients. Thorax 2001;56:266-71.

34. Han JN, Stegen K, Simkens K, et al. Unsteadiness of breathing in patients with hyperventilation syndrome and anxiety disorders. Eur Respir J 1997;10:167-76.

35. Herland K, Akselsen JP, Skjønsberg OH, et al. How representative are clinical study patients with asthma or COPD for a larger "real life" population of patients with obstructive lung disease? Respir Med 2005;99:11-9. 